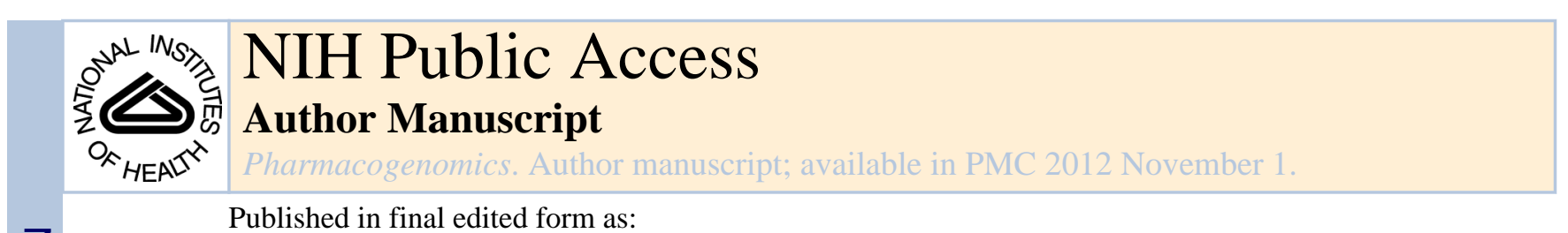

Published in final edited form as:

Pharmacogenomics. 2012 January ; 13(1): 113-121. doi:10.2217/pgs.11.132.

\title{
Exploration of CYP450 and drug transporter genotypes and correlations with nevirapine exposure in Malawians
}

\author{
Kevin C Brown, \\ University of North Carolina at Chapel Hill, Eshelman School of Pharmacy, 3202 Kerr Hall, \\ Chapel Hill, NC 27599-7569, USA \\ Mina C Hosseinipour, \\ University of North Carolina at Chapel Hill, School of Medicine, Chapel Hill, NC, USA and UNC \\ Project, Lilongwe, Malawi \\ Janelle M Hoskins, \\ University of North Carolina at Chapel Hill, Eshelman School of Pharmacy, 3202 Kerr Hall, \\ Chapel Hill, NC 27599-7569, USA and UNC Institute of Pharmacogenomics \& Individualized \\ Therapy, Chapel Hill, NC, USA \\ Ranjit K Thirumaran, \\ St Jude Children's Research Hospital, Department of Pharmaceutical Sciences, Mempis, TN, \\ USA
}

\section{Hsiao-Chuan Tien, \\ UNC FPG Child Development Institute, Chapel Hill, NC, USA}

\author{
Ralf Weigel, \\ Lighthouse Clinic, Lilongwe, Malawi
}

Jean Tauzie,

Lighthouse Clinic, Lilongwe, Malawi

Ida Shumba,

Lighthouse Clinic, Lilongwe, Malawi

Jatinder K Lamba,

University of Minnesota, College of Pharmacy, Minneapolis, MN, USA and University of

Minnesota, Institute of Human Genetics, Minneapolis, MN, USA

\section{Erin G Schuetz,}

\footnotetext{
(C) 2012 Future Medicine Ltd.

*Author for correspondence: University of North Carolina at Chapel Hill, Eshelman School of Pharmacy, 3202 Kerr Hall, Chapel Hill, NC 27599-7569, USA, Tel.: +1 919843 2280, Fax: +1 919962 0644, amanda_corbett@unc.edu.

Ethical conduct of research

The authors state that they have obtained appropriate institutional review board approval or have followed the principles outlined in the Declaration of Helsinki for all human or animal experimental investigations. In addition, for investigations involving human subjects, informed consent has been obtained from the participants involved.

For reprint orders, please contact: reprints@futuremedicine.com

Financial \& competing interests disclosure

This study was supported in part by the Pharmacogenetics for Every Nation Initiative, the Pharmacogenetics Research Network (NIH U01 GM63340), the UNC Translational and Clinical Sciences Institute (UL1RR025747), NIH Grant GM60346, Cancer Center Support Grant (NIH P30 CA21765), and by the American Lebanese Syrian Associated Charities (ALSAC). The authors have no other relevant affiliations or financial involvement with any organization or entity with a financial interest in or financial conflict with the subject matter or materials discussed in the manuscript apart from those disclosed.

No writing assistance was utilized in the production of this manuscript.
} 
St Jude Children's Research Hospital, Department of Pharmaceutical Sciences, Mempis, TN, USA

\title{
Howard L McLeod,
}

University of North Carolina at Chapel Hill, Eshelman School of Pharmacy, 3202 Kerr Hall, Chapel Hill, NC 27599-7569, USA and UNC Institute of Pharmacogenomics \& Individualized Therapy, Chapel Hill, NC, USA

Angela DM Kashuba, and University of North Carolina at Chapel Hill, Eshelman School of Pharmacy, 3202 Kerr Hall, Chapel Hill, NC 27599-7569, USA and University of North Carolina at Chapel Hill, Center for AIDS Research, Chapel Hill, NC, USA

\section{Amanda H Corbett ${ }^{\star}$ University of North Carolina at Chapel Hill, Eshelman School of Pharmacy, 3202 Kerr Hall, Chapel Hill, NC 27599-7569, USA}

\section{Abstract}

Aim-Genetic polymorphisms have the potential to influence drug metabolism and vary among ethnic groups. This study evaluated the correlation of genetic polymorphisms with nevirapine pharmacokinetics exposure in Malawians.

Materials \& methods-CYP450 2B6, 2D6, $3 A 4$ and $3 A 5, A B C B 1$ and constitutive androstane receptor and pregnane $\mathrm{X}$ receptor, were analyzed for polymorphisms in 26 subjects.

\begin{abstract}
Results-Allele frequencies (variant) were: CYP2B6 514G>T (0.31) CYP2D6*4 (0.02); CYP2D6*17 (0.35); CYP3A4*1B (0.77); CYP3A5*3 (0.25); ABCB1 2677G>T (0.0), ABCB1 $3435 \mathrm{C}>\mathrm{T}(0.21), N R 11313711152 \mathrm{~T}>\mathrm{C}(0.02), N R 1 I 244477 \mathrm{~T}>\mathrm{C}(0.10), N R 11263396 \mathrm{C}>\mathrm{T}(0.33)$, NRII2 6-bp indel (del: 0.17). CYP2B6 516G>T (non-wild-type/wild-type) correlated with nevirapine pharmacokinetic parameters; geometric mean ratios (95\% CI): $1.75(1.27-2.40)$ for area under the concentration time curve (AUC) $)_{0-12} \mathrm{~h}, 1.58$ (1.03-2.42) for $\mathrm{C}_{0}$, and $0.53(0.31-$ 0.91 ) for clearance. In a multivariable model, nevirapine AUC increased by $1.5 \%$ per year of age ( $\mathrm{p}<0.0001), C Y P 2 B 6516 \mathrm{~T}$ allele increased AUC by $92 \%(\mathrm{p}<0.0001)$, and $C Y P 3 A 5 * 3$ decreased AUC by $31 \%(\mathrm{p}=0.0027)$.
\end{abstract}

Conclusion-Allele frequencies were similar to other sub-Saharan African populations. The $\mathrm{T}$ allele for CYP2B6 516 was significantly associated with nevirapine exposure.

\section{Keywords}

CYP2B6; CYP450; Malawi; nevirapine; nuclear receptor; P-glycoprotein; pharmacokinetics

Genetic polymorphisms have been identified in genes that encode drug-metabolizing enzymes and transporters such as the CYP450s (CYPs; CYPs) and P-glycoproteins (MDR1; $A B C B 1$ ) [1]. These polymorphisms may explain interindividual differences in drug pharmacokinetics [1]. Patients with genotypes that produce less active enzymes in major drug metabolic pathways have reduced metabolism and therefore higher drug exposures and potentially more adverse effects. Patients with activity-enhancing genotypes clear drugs faster, which can lead to subtherapeutic concentrations and increased risk of therapy failure [1]. Specifically, $C Y P$ polymorphisms have been associated with interpatient variation in antiretroviral plasma concentrations, and the allele frequencies vary among ethnic groups $[2,3]$.

HIV-infected patients on antiretroviral therapy have demonstrated altered plasma concentrations due to differing CYP450 enzyme genotypes [2,4,5]. These genotypes have 
also shown differing frequencies based on ethnicity [6]. A Ugandan population has been shown to have significantly higher drug exposure compared to western populations; this can partially be explained by different frequencies of genetic polymorphisms in drugmetabolizing enzymes and transporters [7].

Antiviral therapy is expanding in developing countries using doses optimized for western populations. We recently found that steady-state nevirapine concentrations were significantly increased in a group of Malawian adults and children compared to concentrations seen in a mostly western Caucasian cohort of adults and children [8-11]. The 1.6- to 2.2-fold increase in drug exposure could not be explained by weight, age or other observable patient-specific factors. Nevirapine is non-nucleoside reverse transcriptase inhibitor used for the treatment of HIV infections in adults and children. It is metabolized by the CYP450 system, specifically CYP3A4/5, CYP2D6 and CYP2B6 [12], and data suggest that polymorphisms in the genes encoding these enzymes can alter nevirapine concentrations [2,7,13-18]. The clinical impact of the CYP2B6 polymorphism, 516G $>\mathrm{T}$, has been described in various settings. The use of single-dose nevirapine to prevent perinatal vertical transmission has been shown to have prolonged exposures in women with the CYP2B6 516 $\mathrm{T}$ allele, which greatly increases the risk of developing non-nucleoside reverse transcriptase inhibitor resistance mutations [18].

Nevirapine autoinduces its metabolism by activating CYP3A4/5 and CYP2B6 expression, and data show that nevirapine may do this through the activation of the nuclear receptor, constitutive androstane receptor (CAR; NR1I3) [19]. The nuclear receptor pregnane X receptor (PXR; NR1I2) regulates basal expression of CYP3A4/5 [20]. Polymorphisms in the genes coding for these nuclear receptors have been shown to influence the expression of the CYPs and therefore influence nevirapine exposure [19-21].

Frequencies of polymorphisms in CYP3A4/5, CYP2B6, ABCB1, PXR and CAR can vary between ethnic groups [22], which might contribute to the difference in drug exposure we previously observed between Malawians and western subjects [2,8,9]. However, the frequencies of the polymorphisms in the abovementioned genes have not been reported in a Malawian population.

\section{Materials \& methods}

\section{Study design}

This was a nonrandomized pharmacogenetic observational study in HIV-infected Malawian children and adults who participated in pharmacokinetic studies evaluating nevirapine [8,9]. The University of North Carolina Institutional Review Board and the Malawi National Health Sciences Research Committee approved the study protocol. All research subjects provided consent or assent with guardian consent. The objective was to determine the frequencies of $C Y P, A B C B 1$ and nuclear receptor polymorphisms in 30 Malawian adults and children and to explore correlations between their genotypes and nevirapine (Virammune ${ }^{\circledR}$ ) exposure.

\section{Samples \& DNA extraction}

Nevirapine concentrations were measured in blood plasma by a validated HPLC-UV method as previously described $[8,9,23]$.

DNA was isolated from whole blood using the QIAamp ${ }^{\circledR}$ DNA Mini kit (Qiagen Sciences, MD, USA). Genotyping was performed using PCR and Pyrosequencing ${ }^{\circledR}$ as previously described [24]. Primers and PCR conditions for ABCB1 2677 (rs2032582) and 3435 (rs1045642), CYP2B6 516G>T (Q172H; rs3745274), CYP2D6*4 (rs3892097), CYP3A4*1B 
(rs2740574), and CYP3A5*3 (rs776746) were previously described [24-27]. For CYP2D6*17 (rs28371706), DNA was amplified using the forward primer: 5'-GAG GCG CTG GTG ACC CAC- $3^{\prime}$ and biotinylated reverse primers: $5^{\prime}$-biotin-CTG TCC CCA CCG CTG CTT-3' at an annealing temperature of $65^{\circ} \mathrm{C}$. The internal primer was $5^{\prime}$-CGC CTG TGC CCA TCA- $3^{\prime}$ and the sequence to analyze was C/TCCAGATCC.

NR1I2 44477T >C (rs1523130), 63396C > T (rs2472677) and the 6-bp deletion (6-bp indel; rs3842689) were genotyped by previously described methods [20]. The trans NR1I3 polymorphism, rs10494390 (NT_004487.18:g.13711152T>C), was amplified from $50 \mathrm{ng}$ of genomic DNA by the forward 5'-ACCTTCGGGGTTGAATTTT-3' and reverse 5'TGTAGCATTTGGGTTTTGGA-3' primers.

\section{Data analysis/statistics}

Genotype data from the Malawi sample was compared to genotype data of Yoruban (subSaharan African), African-American and Caucasians of European descent obtained from the HapMap database. Fisher's Exact Test was used to determine whether the Malawian sample's genotype frequencies differed significantly from the archived data. The CochranArmitage Trend Test was employed to determine if a genotype trend could be detected between groups.

The pharmacokinetic parameters were generated by noncompartmental analysis using WinNonlin (v5.2, Pharsight, Inc., NC, USA). Area under the concentration time curve (AUC) $0-12 \mathrm{~h}$ was extrapolated from partial dosing interval data (adults: $0-8 \mathrm{~h}$, children: 0-6 h). Weight-adjusted $(\mathrm{kg})$ apparent oral clearance $(\mathrm{CL} / \mathrm{F})$ was determined to confirm AUC results of the pediatric subjects. Trough concentrations $\left(\mathrm{C}_{0}\right)$ are predose concentrations. Statistical analysis was performed using SAS (v9.1.3, SAS Institute, NC, USA). Geometric mean ratios (GMRs) of non-wild-type (WT) versus WT genotypes were calculated using linear regression of natural $\log$ transformed pharmacokinetic parameters with $\alpha$ significance levels of $5 \%$ and $0.625 \%$; the latter to account for multiple statistical tests for the eight genotypes tested. Multiple linear regression was performed to develop a multiple variable model $(\alpha=0.05)$ with AUC as outcome and genotypes and demographics as covariates. WT genotypes are defined as genotypes that are homozygous for the WT allele, and non-WT genotypes are defined as genotypes that are either heterozygous or homozygous for the variant (VAR) allele. WT alleles are defined by having functional enzyme gene products, often designated as $* l$ for the CYPs, or having the highest allele frequency in this study sample.

\section{Results}

DNA was isolated from 24 Malawian subjects, of whom 11 were adults with a median (range) age of 40 (26-46) years, weight of $72(60-80) \mathrm{kg}$, BMI of $25.4(19.8-32.5) \mathrm{kg} / \mathrm{m}^{2}$ and five were male. Thirteen were children with median (range) age of 6.7 (1.3-13.6) years, weight of 18 (9-30.5) kg, BMI z-score of $0.31(-5.39-1.28)$ and six were male [8,9].

Subjects were also taking trade formulations of lamivudine (Epivir ${ }^{\circledR}$ ) and stavudine (Zerit ${ }^{\circledR}$ ) at the time of pharmacokinetic sampling. Nevirapine was dosed using standard dosing in adults at $200 \mathrm{mg}$ twice daily. The pediatric group was dosed using standard weight-based dosing, in which children up to 8 years of age are dosed $7 \mathrm{mg} / \mathrm{kg}$ twice daily and children 8 years of age and older are dose $4 \mathrm{mg} / \mathrm{kg}$ twice daily.

Genotype frequencies of 26 Malawians are presented in Table 1; genetic sampling included two subjects without pharmacokinetic data. The genotypes could not be reliably tested for Hardy-Weinberg equilibrium due to a small sample size. The Malawian genotype frequencies did not differ from Yorubans. The Malawian's CYP2D6*17 and $A B C B 1$ 
2677G $>$ T genotype data differed from that of the African-American individuals; other genotypes tested did not differ. The Malawian genotype frequencies differed from Caucasians in regards to all genotypes tested except for CYP2B6 516G $>\mathrm{T}(\mathrm{p}=0.31)$. The VAR allele frequencies of $C Y P 3 A 4 * 1 B$ and $C Y P 2 D 6 * 17$ were higher and $C Y P 3 A 5 * 3$, CYP2D6*4, ABCB1 2677G > T, ABCB1 3435C > T, NR113 13711152T >C, NR112 44477T >C, $63396 \mathrm{C}>\mathrm{T}$ and 6-bp indel were lower in the Malawians than Caucasians.

The results of the correlation analysis are listed in Table 2 . The pharmacokinetic data were $\log$ normally distributed. With $\alpha=0.05$, the CYP2B6 $516 \mathrm{~T}$ allele was significantly associated with increased $\mathrm{AUC}, \mathrm{C}_{0}$ and $\mathrm{CL} / \mathrm{F}$. With $\alpha=0.00625$ for multiple comparison correction, this significance was lost . The NR112 6-bp indel deletion allele was significantly associated with decreased $\mathrm{AUC}_{0-12 \mathrm{~h}}$ and increased CL/F with $\alpha=0.05$; however, this lost significance with $\alpha=0.00625$.

Figure 1 shows a decrease in weight-based $\mathrm{CL} / \mathrm{F}$ with the number of $C Y P 2 D 6 * 17$ alleles within the pediatric group. This was not seen in the adult subjects (Figure 1b). In order to confirm the effect of $C Y P 2 D 6 * 17$ on $\mathrm{CL} / \mathrm{F}$ in the pediatric group, the GMR for CL/F was determined separately in the pediatric and adult groups. The GMR (VAR/WT; 95\% CI) for CYP2D6*17 was $0.59(0.36-0.98 ; \mathrm{p}=0.04)$ and $1.02(0.61-1.70 ; \mathrm{p}=0.9)$ for children and adults, respectively, demonstrating that $C Y P 2 D 6^{*} 17$ is associated with decreased clearance only in the pediatric group of our sample.

The following multiple variable model was determined for nevirapine AUC: $\operatorname{Ln}\left(\mathrm{AUC}_{0-12 \mathrm{~h}}\right)$ $=10.67+0.65(2 \mathrm{~B} 6 \mathrm{Var})-0.37(3 \mathrm{~A} 5 \mathrm{Var})+0.015$ (age). The CYP2B6 $516 \mathrm{~T}$ allele increases $\mathrm{AUC}_{0-12 \mathrm{~h}}$ by $92 \%$ (95\% CI: $51-145 \%$ ), CYP $3 A 5 * 3$ allele decreases $\mathrm{AUC}_{0-12 \mathrm{~h}}$ by $31 \%$ (95\% CI: $12-46 \%$ ) and age increases $\mathrm{AUC}_{0-12 \mathrm{~h}}$ by $1.5 \%$ (95\% CI: $0.7-2 \%$ ) per year. Table 3 shows the p-values for each of the variables tested. The effect of the PXR 6-bp indel on nevirapine AUC was not significant in the final multiple variable model despite significance $(\alpha=0.05)$ seen in the univariate analysis. A similar model was determined for weightadjusted CL/F (not shown).

\section{Discussion}

CYP2B6 genotype is associated with intra-population differences in Malawians. Consistent with previous studies [7], the T allele at CYP2B6 516 correlated with increased exposure and decreased clearance of nevirapine. Since our study and others have demonstrated association of the $\mathrm{T}$ allele with increased nevirapine exposures and varying frequencies of $C Y P$ polymorphisms among ethnic groups, antiretrovirals must be evaluated in all potential treatment populations $[2,7]$.

Drug-metabolizing enzyme and transporter genotype data from our sample agree with other African populations as reported in the HapMap database (Luhya in Webuye, Kenya; Maasai in Kinyawa, Kenya and the Yoruban in Ibadan, Nigeria) [22]. For example, CYP2B6 $516 \mathrm{~T}$ allele frequencies ranged from 0.315 to 0.42 for the African groups in the HapMap database; the Malawian frequency was 0.31 . For $C Y P 3 A 5 * 3$, the HapMap database reports a wide range of allele frequencies: $0.128-0.486$ (Malawians frequency: 0.25 ), which demonstrates sub-Saharan African ethnic groups could have dissimilar CYP allele frequencies. This may be clinically relevant to drugs metabolized by these CYP enzymes.

Following the multiple comparisons correction, CYP2B6 $516 \mathrm{~T}$ allele correlated with increased AUC. CYP2B6 and age were independently associated with nevirapine AUC, $\mathrm{C}_{0}$ and $\mathrm{CL} / \mathrm{F}$. $C Y P 3 A 5 * 3$ is an allele that causes decreased function of $C Y P 3 A 5$ and is expected to decrease clearance of nevirapine, thereby increasing its exposure. Although there was no correlation in the univariate analysis, $C Y P 3 A 5 * 3$ was associated with decreased AUC when 
controlling for $C Y P 2 B 6$ and age. $C Y P 3 A 5 * 3$ correlating with lower nevirapine exposure may be a result of heterozygous individuals in our sample expressing more enzyme than homozygous WT individuals and/or CYP $3 A 5 * 3$ being in linkage with another functional allele [28]. In addition, our sample did not include individuals who were $C Y P 3 A 5 * 3 / * 3$, which is a genotype found only in Africans based on the HapMap data, which may introduce bias in our model.

The $C Y P 2 D 6 * 17$ allele appears to be specific to African populations and is associated with decreased CYP2D6 enzyme activity [3]. Only within the pediatric group did CYP2D6*17 correlate with a decrease in weight-adjusted nevirapine CL/F, suggesting that CYP2D6 may play a larger role in nevirapine metabolism in children. However, this would need to be confirmed in a larger study population accounting for other CYP2D6 SNPs and other polymorphisms in linkage disequilibrium.

CAR and PXR are functionally linked with the expression of various CYPs [19]. Nevirapine preferentially induces CYP2B6 through activation of CAR [19], and polymorphisms in PXR are associated with higher basal expression and reduced induction of CYP3A4 activity [20]. Polymorphisms in NR1I3 and NRII2 may attenuate the induction of CYP2B6 and CYP3A5 leading to higher nevirapine exposures; however, our study did not demonstrate this. The NRII2 6-bp indel, which is in linkage disequilibrium with NR1I2 44477T >C, was associated with lower nevirapine exposures, which may indicate increased basal expression of CYP3A4.

The multivariable model showed that nevi-rapine concentrations were influenced by CYP2B6 and CYP3A5 genotypes in addition to age. Currently there is limited clinical utility for this model, due to a lack of widely available genotypic testing for these enzymes in all populations. In addition, there is limited data linking genotype, concentration and safety and/ or efficacy. Despite this, the clinical implications of this model include that it is possible to predict nevirapine exposures based on genotype. With more advanced modeling methods, nevi-rapine concentrations could be predicted based on genotype and other known patient factors in order to predict adverse effects such as rash due to supratherapeutic concentrations or virologic failure due to subtherapeutic concentrations. However, these models will also be limited to the groups in which they are studied. In addition, this is a potentially useful tool for research purposes in which outliers of drug concentrations exist with no obvious decreased adherence or extra dosing.

The US Department of Health and Human Services (US DHHS) currently recommends a target nevirapine trough plasma concentration of $3 \mu \mathrm{g} / \mathrm{ml}$ based on data demonstrating a five-fold increase in virologic failure risk with lower trough concentrations [29,30,101]. These data were developed in a Caucasian population with the same doses currently being used in African populations. The Malawian and other sub-Saharan African HIV-infected patients would benefit greatly from efficacy and safety studies focusing on their populations since there is an overwhelming amount of data demonstrating that these groups have genetic differences that affect how the drugs are metabolized and eliminated.

Factors such as nutritional status and diet can also affect drug metabolism. Low protein and high carbohydrate consumption are associated with decreased hepatic CYP metabolism, while high protein diets are associated with increased metabolism [31]. Our subjects had normal nutritional status based on BMI, except for one underweight child. Western diets are generally higher in animal protein than those in sub-Saharan Africa, while diets in Malawi are considerably lower in animal protein and include carbohydrate-rich protein sources [102]. This dietary factor may contribute to the differences observed in nevirapine exposure between Malawians and westerners. 
Despite our limited sample size, our novel pilot data provide evidence that ethnic diversity of drug-metabolizing enzyme genetics may play a role in antiretroviral drug exposures. Larger studies are needed to define population allele frequencies and to evaluate the clinical impact of drug-metabolizing enzymes, transporter and nuclear receptor polymorphisms.

\section{References}

Papers of special note have been highlighted as:

- of interest

-" of considerable interest

1. Evans WE, McLeod HL. Pharmacogenomics - drug disposition, drug targets, and side effects. N Engl J Med. 2003; 348(6):538-549. [PubMed: 12571262]

2--. Rotger M, Colombo S, Furrer H, et al. Influence of CYP2B6 polymorphism on plasma and intracellular concentrations and toxicity of efavirenz and nevirapine in HIV-infected patients. Pharmacogenet Genomics. 2005; 15(1):1-5. First paper to describe CYP2B6 polymorphisms and effects on nevirapine therapy. [PubMed: 15864119]

3. Bradford LD. CYP2D6 allele frequency in European Caucasians, Asians, Africans and their descendants. Pharmacogenomics. 2002; 3(2):229-243. [PubMed: 11972444]

4. Haas DW, Wu H, Li H, et al. MDR 1 gene polymorphisms and phase 1 viral decay during HIV-1 infection: an adult aids clinical trials group study. J Acquir Immune Defic Syndr. 2003; 34(3):295298. [PubMed: 14600574]

5. Haas DW, Ribaudo HJ, Kim RB, et al. Pharmacogenetics of efavirenz and central nervous system side effects: an adult aids clinical trials group study. AIDS. 2004; 18(18):2391-2400. [PubMed: 15622315]

6. Klein K, Lang T, Saussele T, et al. Genetic variability of CYP2D6 in populations of African and Asian origin: allele frequencies, novel functional variants, and possible implications for anti-HIV therapy with efavirenz. Pharmacogenet Genomics. 2005; 15(12):861-873. [PubMed: 16272958]

7-•. Penzak SR, Kabuye G, Mugyenyi P, et al. Cytochrome p450 2B6 (CYP2B6) G516T influences nevirapine plasma concentrations in HIV-infected patients in Uganda. HIV Med. 2007; 8(2):8691. First paper to present nevirapine concentration differences associated with $C Y P 2 B 6$ genotype in a group of Africans. [PubMed: 17352764]

8-. Hosseinipour MC, Corbett AH, Kanyama C, et al. Pharmacokinetic comparison of generic and trade formulations of lamivudine, stavudine and nevirapine in HIV-infected Malawian adults. AIDS. 2007; 21(1):59-64. Describes the adult pharmacokinetic study on which this present pharmacogenetic study was based. [PubMed: 17148968]

9-. Corbett AH, Hosseinipour MC, Nyirenda J, et al. Pharmacokinetics of generic and trade formulations of lamivudine, stavudine and nevirapine in HIV-infected Malawian children. Antiviral Ther. 2010; 15(1):83-90. Describes the pediatric pharmacokinetic study on which this present pharmacogenetic study was based.

10. van Heeswijk RP, Veldkamp AI, Mulder JW, et al. The steady-state pharmacokinetics of nevirapine during once daily and twice daily dosing in HIV-1-infected individuals. AIDS. 2000; 14(8):F77-F82. [PubMed: 10853971]

11. Luzuriaga K, Bryson Y, McSherry G, et al. Pharmacokinetics, safety, and activity of nevirapine in human immunodeficiency virus type 1-infected children. J Infect Dis. 1996; 174(4):713-721. [PubMed: 8843207]

12. Erickson DA, Mather G, Trager WF, Levy RH, Keirns JJ. Characterization of the in vitro biotransformation of the HIV-1 reverse transcriptase inhibitor nevirapine by human hepatic cytochromes P-450. Drug Metab Disp. 1999; 27(12):1488-1495.

13. Wyen C, Hendra H, Vogel M, et al. Impact of CYP2B6 983T $>\mathrm{C}$ polymorphism on non-nucleoside reverse transcriptase inhibitor plasma concentrations in HIV-infected patients. J Antimicrob Chemother. 2008; 61(4):914-918. [PubMed: 18281305] 
14--. Saitoh A, Sarles E, Capparelli E, et al. CYP2B6 genetic variants are associated with nevirapine pharmacokinetics and clinical response in HIV-1-infected children. AIDS. 2007; 21(16):21912199. First paper to present nevirapine concentration differences associated with $C Y P 2 B 6$ genotype in children. [PubMed: 18090046]

15. Ramachandran G, Ramesh K, Hemanth Kumar AK, et al. Association of high T allele frequency of CYP2B6 G516T polymorphism among ethnic south Indian HIV-infected patients with elevated plasma efavirenz and nevirapine. J Antimicrob Chemother. 2009; 63(4):841-843. [PubMed: 19218571]

16. Mahungu T, Smith C, Turner F, et al. Cytochrome p450 2B6 516G-->T is associated with plasma concentrations of nevirapine at both $200 \mathrm{mg}$ twice daily and $400 \mathrm{mg}$ once daily in an ethnically diverse population. HIV Med. 2009; 10(5):310-317. [PubMed: 19228205]

17. Haas DW, Gebretsadik T, Mayo G, et al. Associations between CYP2B6 polymorphisms and pharmacokinetics after a single dose of nevirapine or efavirenz in African-Americans. J Infect Dis. 2009; 199(6):872-880. [PubMed: 19239339]

18-. Chantarangsu S, Cressey TR, Mahasirimongkol S, et al. Influence of $C Y P 2 B 6$ polymorphisms on the persistence of plasma nevirapine concentrations following a single intra-partum dose for the prevention of mother to child transmission in HIV-infected Thai women. J Antimicrob Chemother. 2009; 64(6):1265-1273. Presents a major public health concern regarding CYP2B6 polymorphisms and the use of single-dose nevirapine for preventing vertical transmission, which is currently a standard practice in many African nations. [PubMed: 19812066]

19. Faucette SR, Zhang TC, Moore R, et al. Relative activation of human pregnane $\mathrm{x}$ receptor versus constitutive androstane receptor defines distinct classes of CYP2B6 and CYP3A4 inducers. J Pharmacol Exp Ther. 2007; 320(1):72-80. [PubMed: 17041008]

20. Lamba J, Lamba V, Strom S, Venkataramanan R, Schuetz E. Novel single nucleotide polymorphisms in the promoter and intron 1 of human pregnane $\mathrm{X}$ receptor/NRII2 and their association with CYP3A4 expression. Drug Metab Disp. 2008; 36(1):169-181.

21. Lamba J, Lamba V, Schuetz E. Genetic variants of PXR (NR1I2) and CAR (NR1I3) and their implications in drug metabolism and pharmacogenetics. Curr Drug Metab. 2005; 6(4):369-383. [PubMed: 16101575]

22-. International HapMap Consortium. The international HapMap project. Nature. 2003; 426(6968): 789-796. This group hosts an online database, which is the most extensive source for genotyping data among different ethnic groups. [PubMed: 14685227]

23. Rezk NL, Tidwell RR, Kashuba AD. Simultaneous determination of six HIV nucleoside analogue reverse transcriptase inhibitors and nevirapine by liquid chromatography with ultraviolet absorbance detection. J Chromatog. 2003; 791(1-2):137-147.

24. Marsh S, King CR, Garsa AA, McLeod HL. Pyrosequencing of clinically relevant polymorphisms. Methods Mol Biol (Clifton, NJ). 2005; 311:97-114.

25. Garsa AA, McLeod HL, Marsh S. CYP3A4 and CYP3A5 genotyping by pyrosequencing. BMC Med Genet. 2005; 6:19. [PubMed: 15882469]

26. Rohrbacher M, Kirchhof A, Geisslinger G, Lötsch J. Pyrosequencing-based screening for genetic polymorphisms in cytochrome P450 2B6 of potential clinical relevance. Pharmacogenomics. 2006; 7(7):995-1002. [PubMed: 17054410]

27. Zackrisson AL, Lindblom B. Identification of $C Y P 2 D 6$ alleles by single nucleotide polymorphism analysis using pyrosequencing. Eur J Clin Pharmacol. 2003; 59(7):521-526. [PubMed: 13680033]

28. Kuehl P, Zhang J, Lin Y, et al. Sequence diversity in CYP3A promoters and characterization of the genetic basis of polymorphic CYP3A5 expression. Nat Genet. 2001; 27(4):383-391. [PubMed: 11279519]

29. De Vries-Sluijs TE, Dieleman JP, Arts D, et al. Low nevirapine plasma concentrations predict virological failure in an unselected HIV-1-infected population. Clin Pharmacokinetics. 2003; 42(6):599-605.

30. Veldkamp AI, Weverling GJ, Lange JM, et al. High exposure to nevirapine in plasma is associated with an improved virological response in HIV-1-infected individuals. AIDS. 2001; 15(9):10891095. [PubMed: 11416710] 
31. Ensom, MHH.; Blouin, RA. Dietary influences on drug disposition. In: Burton, ME.; Shaw, LM.; Schentag, JJ.; Evans, WE., editors. Applied Pharmacokinetics and Pharmacodynamics: Principles of therapeutic drug monitoring. Lippincott Williams and Wilkins; Baltimore, MA, USA: 2006. p. 242-256.

\section{Websites}

101. Panel on antiretroviral guidelines for adults and adolescents. Guidelines for the use of antiretroviral agents in HIV-1-infected adults and adolescents. Department of health and human services; January 10. $2011 \mathrm{p}$. 1-166.www.Aidsinfo.Nih.Gov/contentfiles/adultandadolescentgl.Pdf

102. FAO Malawi nutrition profile. Nutrition and Consumer Protection Division. Food and Agriculture Organization of the United Nations; 2008. www.fao.org/ag/agn/nutrition/mwi_en.stm

103. NCBI dbSNP. www.ncbi.nlm.nih.gov/snp 


\section{Executive summary}

\section{Nevirapine pharmacokinetics in Malawians}

- Previously studied HIV-infected adults and children had 1.6- to 2-fold higher exposures compared to published data in western cohorts.

- Drug exposures can be influenced by polymorphisms in genes encoding enzymes, transporters and receptors that are involved in drug metabolism and elimination.

Aim

- We sought to identify the allele frequencies of known genetic polymorphisms in drug-metabolizing enzymes, transporters and nuclear receptor genes in Malawians and evaluate the influence of these genetic polymorphisms on nevirapine pharmacokinetics.

\section{Allele frequency $\&$ genotype correlation with nevirapine pharmacokinetics}

- Malawian allele frequencies were similar to other sub-Saharan Africans, but differed from Caucasians.

- The CYP2B6 $516 \mathrm{~T}$ allele was significantly correlated with increased nevirapine exposures.

- $\quad C Y P 2 D 6 * 17$ was associated with increased clearance only in children.

- In a multivariable model, nevirapine AUC increased by $1.5 \%$ with each year increase in age, increased by $92 \%$ with the presence of the CYP $2 B 6516 \mathrm{~T}$ allele and decreased by $31 \%$ with the presence of $C Y P 3 A 5 * 3$.

\section{Conclusion}

- Genetic polymorphisms influence nevirapine metabolism in Malawians.

- Larger studies are needed to evaluate the clinical impact of genetic variability among ethnic groups. 


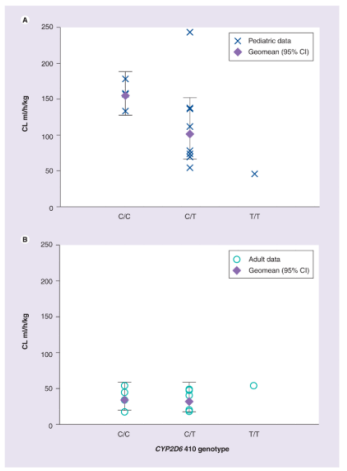

Figure 1. Weight-adjusted clearance versus $C Y P 2 D 6 * 17$ genotype $(* 17=\mathrm{T}$ allele $)$ in the pediatric group and adult group

(A) Pediatric group, (B) Adult group.

CL: Clearance. 


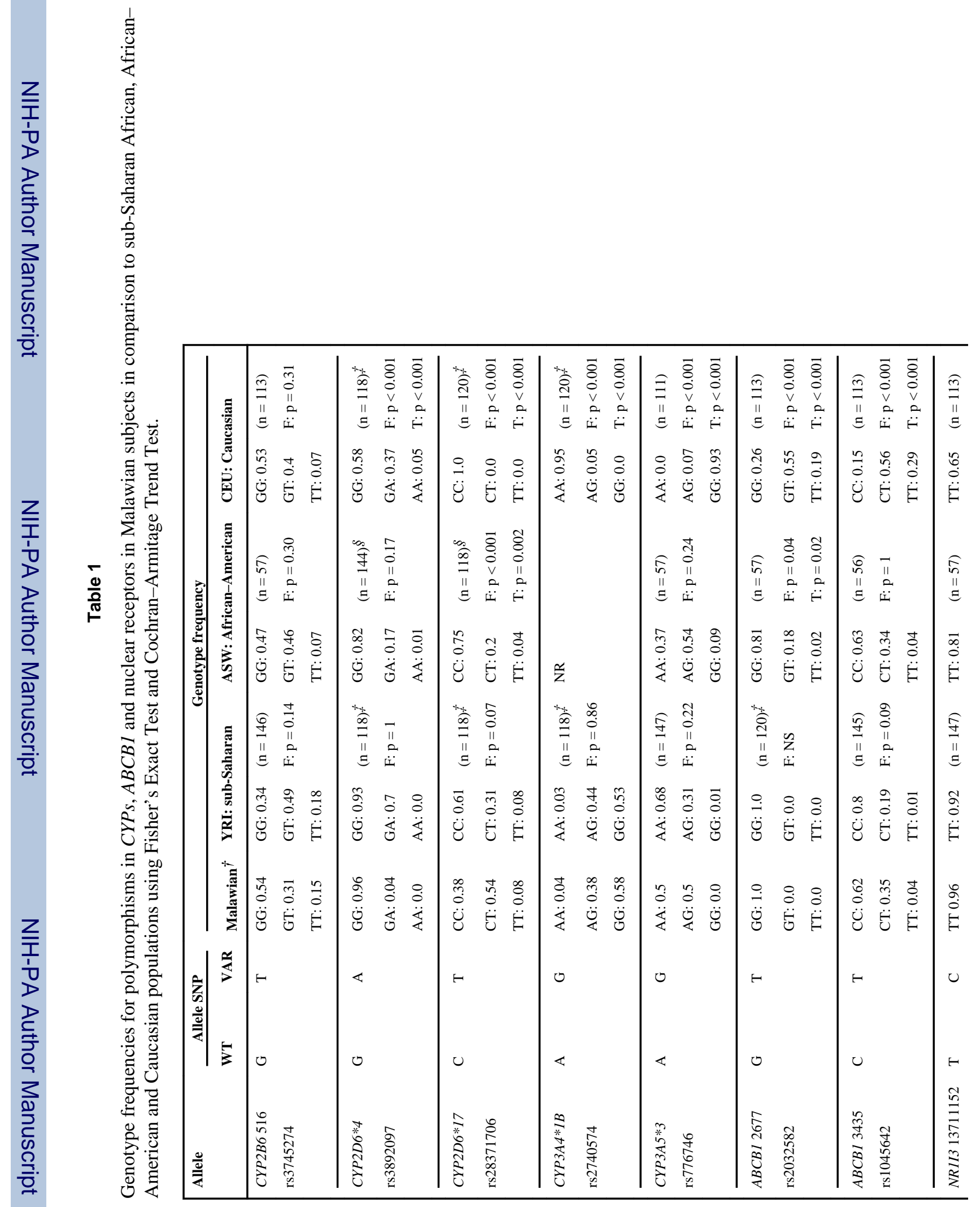




\begin{tabular}{|c|c|c|c|c|c|c|c|c|c|c|c|c|c|}
\hline 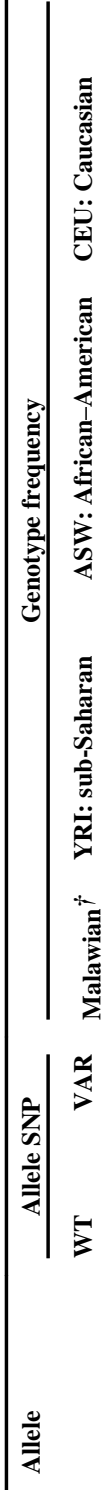 & 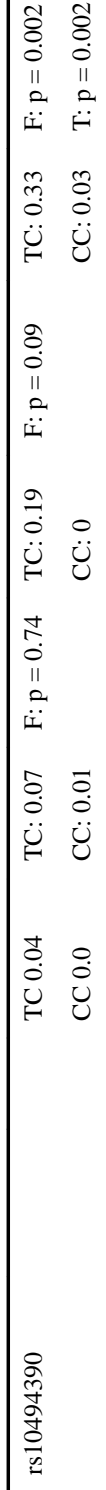 & 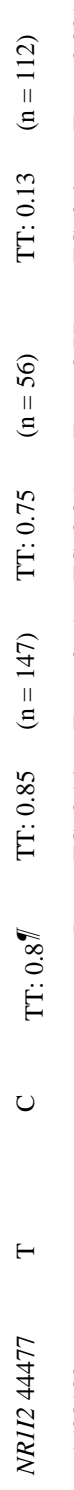 & 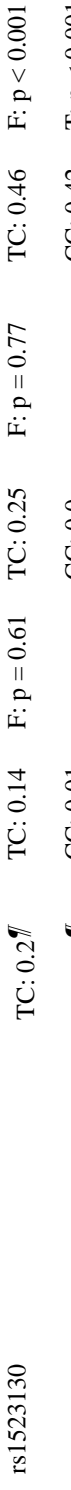 & $\begin{array}{l} \\
\overline{0} \\
\ddot{0} \\
\ddot{u} \\
\overline{0} \\
\dot{0} \\
\ddot{u}\end{array}$ & 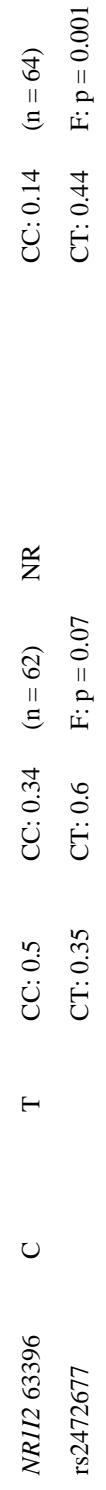 & 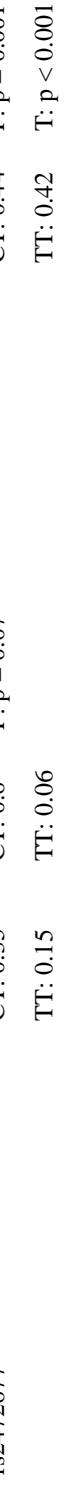 & 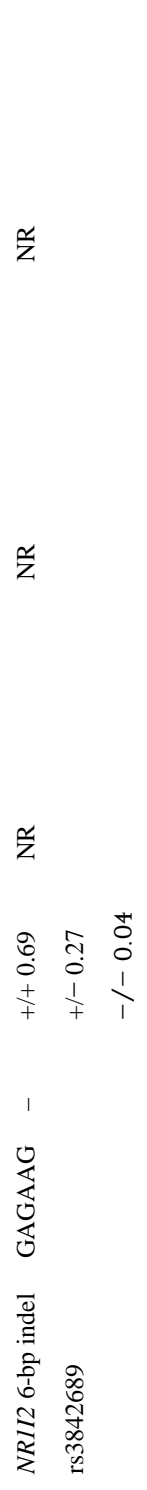 & בี & & 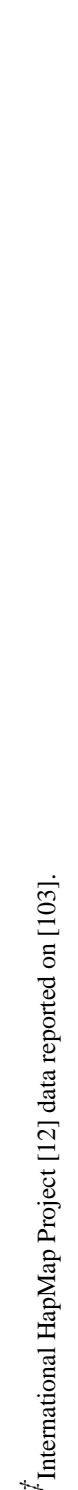 & 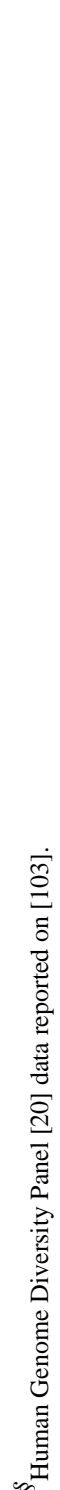 & & 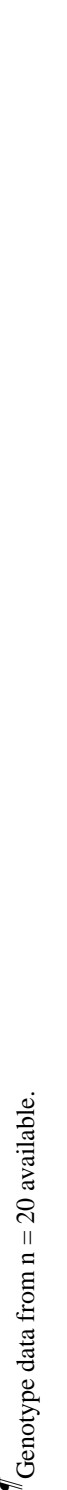 \\
\hline
\end{tabular}


Table 2

Pharmacokinetic parameter geometric mean ratios (non-wild-type or variant versus wild-type).

\begin{tabular}{|c|c|c|c|c|}
\hline Polymorphism & NVP AUC $C_{0-12 ~ h}$ & $\operatorname{NVP} A^{\prime} C_{0-6} \mathbf{h}^{\dagger}$ & $\mathrm{NVPC}_{0}$ & $\mathrm{CL} / \mathrm{F}^{*}$ \\
\hline CYP2B6 516G $>\mathrm{T}$ & $1.75^{*}$ & $1.71^{*}$ & $1.58^{* *}$ & $0.53^{* *}$ \\
\hline rs3745274 & $(1.27-2.40)$ & $(1.25-2.34)$ & $(1.03-2.42)$ & $(0.31-0.91)$ \\
\hline$C Y P 3 A 4 * 1 B^{\S}$ & 0.86 & 0.87 & 0.87 & 0.96 \\
\hline rs2740574 & $(0.59-1.27)$ & $(0.60-1.27)$ & $(0.55-1.39)$ & $(0.53-1.74)$ \\
\hline CYP $3 A 5 * 3$ & 0.91 & 0.92 & 0.93 & 1.03 \\
\hline rs776746 & $(0.62-1.33)$ & $(0.63-1.35)$ & $(0.59-1.48)$ & $(0.57-1.86)$ \\
\hline$C Y P 2 D 6 * 17$ & 1.13 & 1.11 & 1.06 & 0.94 \\
\hline rs28371706 & $(0.76-1.68)$ & $(0.75-1.64)$ & $(0.65-1.70)$ & $(0.51-1.72)$ \\
\hline$A B C B 13435 \mathrm{C}>\mathrm{T}$ & 1.10 & 1.07 & 0.98 & 0.83 \\
\hline rs 1045642 & $(0.75-1.63)$ & $(0.73-1.58)$ & $(0.61-1.57)$ & $(0.46-1.50)$ \\
\hline$N R 11244477 \mathrm{~T}>\mathrm{C}$ & 0.80 & 0.81 & 0.90 & 1.21 \\
\hline rs1523130 & $(0.50-1.28)$ & $(0.51-1.27)$ & $(0.51-1.59)$ & $(0.58-2.49)$ \\
\hline$N R 11263396 \mathrm{C}>\mathrm{T}$ & 0.80 & 0.79 & 0.82 & 1.35 \\
\hline rs2472677 & $(0.55-1.17)$ & $(0.55-1.14)$ & $(0.52-1.30)$ & $(0.76-2.42)$ \\
\hline NRII2 6-bp indel & $0.67^{* *}$ & 0.71 & 0.66 & $1.86^{* *}$ \\
\hline rs3842689 & $(0.45-0.999)$ & $(0.48-1.05)$ & $(0.41-1.07)$ & $(1.02-3.39)$ \\
\hline
\end{tabular}

The geometric mean ratios $(95 \% \mathrm{CI})$ for nevirapine $\mathrm{AUC}_{0}-12 \mathrm{~h}, \mathrm{AUC} 0-6 \mathrm{~h}, \mathrm{C}_{0}$ and $\mathrm{CL} / \mathrm{F}$ comparing data from subjects $(\mathrm{n}=24)$ with at least one variant allele to subjects with wild-type alleles for each SNP. ABCB1 2766G>T was not evaluated because all subjects were homozygous wildtype. CYP2D6*4 and CAR $13711152 \mathrm{C}>\mathrm{T}$ were not evaluated because only one subject had the variant allele.

$\mathrm{p}<0.01$

$* *$

$\mathrm{p}<0.05$.

${ }^{\dagger}$ Partial AUC from 0-6 h for verifying extrapolated AUC $0-12 \mathrm{~h}$.

${ }^{*}$ Weight-adjusted apparent oral clearance.

$\S$ Correlation analysis was performed using $* 1 \mathrm{~B} / * 1 \mathrm{~B}$ versus $* 1 / * 1 \mathrm{~B}$ and $* 1 / * 1$ as only one subject was $* 1 / * 1$.

AUC: Area under the concentration time curve; CL/F: Apparent oral clearance; NVP: Nevirapine. 
Table 3

Multiple variable model determination.

\begin{tabular}{|lll|}
\hline Covariates & p-value & Model \\
\hline$C Y P 2 B 6516 \mathrm{G}>\mathrm{T}$ & 0.0001 & $\mathrm{Y}$ \\
\hline$C Y P 3 A 4 * 1 B$ & 0.5101 & $\mathrm{~N}$ \\
\hline$C Y P 3 A 5 * 3$ & 0.0128 & $\mathrm{Y}$ \\
\hline CYP2D6*17 & 0.237 & $\mathrm{~N}$ \\
\hline ABCB1 3435C $>\mathrm{T}$ & 0.6735 & $\mathrm{~N}$ \\
\hline NR112 44477T $>\mathrm{C}$ & 0.8535 & $\mathrm{~N}$ \\
\hline NR112 63396C $>\mathrm{T}$ & 0.3883 & $\mathrm{~N}$ \\
\hline NR112 6-bp indel & 0.3344 & $\mathrm{~N}$ \\
\hline Age & 0.0017 & $\mathrm{Y}$ \\
\hline Weight & 0.7563 & $\mathrm{~N}$ \\
\hline Sex & 0.2216 & $\mathrm{~N}$ \\
\hline Dose & 0.2215 & $\mathrm{~N}$ \\
\hline
\end{tabular}

$\mathrm{N}$ : Excluded from model; Y: Included in model. 